REVISTA MATEMÁTICA de la

Universidad Complutense de Madrid

Volumen 10, número 2: 1997

http://dx.doi.org/10.5209/rev_REMA.1997.v10.n2.17470

\title{
The cost of controlling unstable systems: time irreversible systems.
}

\author{
Jacques-Louis LIONS and Enrique ZUAZUA*
}

Dedicated to Prof. Luiz Adauto Medeiros in his 70th birthday.

\begin{abstract}
We discuss the cost of controlling parabolic equations of the form $y_{t}-\Delta y-k(-\Delta)^{\theta} y=v 1_{\omega}$ in a bounded smooth domain $\Omega$ of $\boldsymbol{R}^{n}$ with homogeneous Dirichlet boundary conditions where $0 \leq \theta<1$ is fixed. The control $v$ acts on the system through the open and non-empty subset $\omega$ of $\Omega$. As $k \rightarrow \infty$ the system becomes more and more unstable. We analyze the dependence of the control $v$ on the parameter $k$ in the context of various different control problems. We show that the norm of the control diverges when the trajectory is driven from an initial state $y^{0} \in L^{2}(\Omega)$ to the null state at any time $T>0$. However, we prove that the control converges to zero when the null initial state $y^{0}=0$ is driven into a ball of an arbitrary radious $\varepsilon>0$ around a given terminal state $y^{1} \in L^{2}(\Omega)$ or when the solution fulfills exactly a finite number of constraints at time $T$.
\end{abstract}

\section{Introduction}

Let us consider a distributed system whose state $y$ is the solution of the following P.D.E. (Partial Differential Equation):

Mathematics Subject Classification: 93B05, 35K05, 35B35.

Servicio Publicaciones Univ. Complutense. Madrid, 1997.

"Supported by grants PB93-1203 of the DGICYT (Spain) and CHRX-

CT94-0471 of the European Union. 


$$
\frac{\partial y}{\partial t}+\mathcal{A}_{k} y=\mathfrak{B} v
$$

where $\mathcal{A}_{k}$ is a differential operator depending on a parameter $k \in \mathbb{R}$ and where $\mathcal{B}$ is an operator which maps the space of controls (denoted by $v$ ) into the space where $\frac{\partial y}{\partial t}+\mathcal{A}_{k} y$ lies.

For the time being the writing of (1.1) is formal. We shall go into very precise hypotheses later on. It suffices for the moment to assume that (1.1) admits a unique solution, provided one adds to (1.1) an initial condition

$$
\left.y\right|_{t=0}=y(0)=y^{0}, y^{0} \text { given }
$$

and provided also one adds boundary conditions that we do not make explicit for the time being.

We are interested in the cost of controllability when $\mathcal{A}_{k}$ becomes less and less stable as $k \rightarrow \infty$.

Let us make things a little bit more precise.

We are given a finite time horizon $T$ and we are given $y^{1}$ in the state space.

The controllability problem cousists in (trying to) driving, by an appropriate choice of $v$, the system from $y^{0}$ to

$$
\mathcal{N}\left(y^{1}\right)=\text { "neighborhood" of } y^{1} .
$$

Actually "neighborhood" will be taken in several forms, which may be remotely related to the topological meaning of neighborhood.

If we take

$$
\mathcal{N}\left(y^{1}\right)=y^{1}
$$

then one deals with exact controllability.

If

$\mathcal{N}\left(y^{1}\right)=y^{1}+\beta B, \beta>0, B=$ unit ball of the state space

then one deals, if $\beta>0$ can be taken arbitrarily small, with approximate controllability. 
We shall also use a weaker notion where

$$
\mathcal{N}\left(y^{1}\right)=\left\{\begin{array}{l}
\text { set of states whose projection on a finite } \\
\text { dimensional space } E \text { equals the projection of } y^{1}
\end{array}\right.
$$

Assuming (a property that we shall check in all the situations studied here) that there exists a control $v$ in a Hilbert space $\mathcal{U}$ such that

$$
y(T ; v) \in \mathcal{N}\left(y^{1}\right)
$$

we then define the cost of the control as

$$
\inf _{v} \frac{1}{2}\|v\|_{u}^{2}
$$

where $v$ spans the set of all elements in $U$ such that (1.7) is satisfied.

The quantity (1.8) is a function of $k, y^{0}, \mathcal{N}\left(y^{1}\right)$ :

$$
\phi\left(k ; y^{0}, \mathcal{N}\left(y^{1}\right)\right)
$$

We shall refer at this type of functions as the cost and we want to study the behaviour of all these functions as $k \rightarrow \infty$ (i.e. as $\mathcal{A}_{k}$ becomes less and less stable).

We shall consider operators $\mathcal{A}_{k}$ with the following structure:

$$
\mathcal{A}_{k}=\mathcal{A}-k \mathcal{C}
$$

where

$$
\begin{gathered}
\mathcal{A} \text { is a linear coercive operator } \\
\left\{\begin{array}{l}
\mathcal{C} \text { is an unbounded operator which is linear, } \\
\text { coercive and strictly weaker than } \mathcal{A} .
\end{array}\right.
\end{gathered}
$$

It follows from (1.11)-(1.12) that the problem (1.1)-(1.2) and the boundary conditions which will be expressed by

$$
y(t) \in \text { Domain of } \mathcal{A}
$$

admit a unique solution. 
Remark 1.1. We can also remark that problem (1.1), (1.2), (1.13) admits a unique solution for all $k \in \mathbb{R}$. The system becomes less and less (resp. more and more) stable as $k \rightarrow+\infty$ (resp. $k \rightarrow-\infty$ ).

We can now preseut what is the bottom line of the paper, and of subsequent papers. A preliminary remark is in order.

Remark 1.2. With hypotheses (1.11)-(1.12), system (1.1) is time irreversible. The backward (in time) problem is not well set but, at least formally, we can expect different results (actually "opposite" results) as $k \rightarrow \infty$ or $k \rightarrow-\infty$, and also as we take $y^{0} \neq 0, y^{1}=0$ or when we take $y^{0}=0, y^{1} \neq 0$.

Then the general form of the results we wish to prove is as follows:

$$
\left\{\begin{array}{c}
\phi\left(k ; y^{0}, 0\right) \rightarrow+\infty \text { as } k \rightarrow+\infty \\
(\text { and } \rightarrow 0 \text { as } k \rightarrow-\infty)
\end{array}\right.
$$

and

$$
\left\{\begin{array}{c}
\phi\left(k ; 0, \mathcal{N}\left(y^{1}\right)\right) \rightarrow 0 \text { as } k \rightarrow+\infty \\
(\text { and } \rightarrow+\infty \text { as } k \rightarrow-\infty)
\end{array}\right.
$$

A few remarks are in order, before we proceed with more precise statements.

Remark 1.3. Very roughly speaking, (1.14) means that it costs more and more to drive a more and more unstable system from $y^{0} \neq 0$ to 0 , and (1.15) means that it costs less and less to drive a more and more unstable system from 0 to $\mathcal{N}\left(y^{1}\right)$.

Remark 1.4. Of course in (1.14) we can replace " 0 " by some kind of "neighborhood" of 0 . This remark will be used in Section 3.3.

Remark 1.5. As we shall make precise in section 2, the controls we consider in this paper are distributed controls. For the case of boundary controls we refer to [LiZ5]. 
Remark 1.6. The situation is different for similar questions in the case of time reversible systems. We shall present this case in a second paper of this series.

Remark 1.7. Behind the above statements there is the general question of comparing couples of operators $\left\{\mathcal{A}_{k}, \mathcal{B}\right\}$, in order to estimate the cost of a couple. When is a couple "better" than another?. Of course one has then to introduce notions independent of $y^{0}$ (or $y^{1}$ ), i.e. to introduce

$$
\sup _{\left\|y^{0}\right\| \leq 1} \phi\left(k ; y^{0}, 0\right)
$$

and

$$
\sup _{\left\|y^{1}\right\| \leq 1} \phi\left(k ; 0, \mathcal{N}\left(y^{1}\right)\right)
$$

Notions of this type bave been introduced in [Li3] but without connections with unstability. We hope to return to these questions.

Remark 1.8. It seems plausible to think that somewhat similar results, at least locally around a trajectory, will hold for some non linear systems with unstability. But this is an open direction of research.

Remark 1.9. A number of numerical experiments concerning the questions studied here are presented in [GLi].

The content of the paper is now as follows.

In section 2 we define in a precise manuer a class of examples which enter in the general above framework.

In section 3 we study the cost of controlling to zero (or near zero), and in Sections 4 and 5 we study the cost of controlling from zero to some "neighborhood" of a given state. 
Some other cases are briefly indicated in Section 6, which can be treated by the quite general methods introduced in Section 3, 4 and 5 .

In Appendix A we give the technical details of the proof of a uniqueness result that is used in section 3. Finally, in Appendix B we give the proof of an abstract result showing that approximate controllability implies simultancous finite-approximate controllability.

\section{A family of distributed systems}

Let $\Omega$ be a bounded smooth domain of $\mathbb{R}^{n}, n \geq 1$.

Given $T>0$ let us consider the following parabolic equation in the cylinder $Q=\Omega \times(0, T)$ :

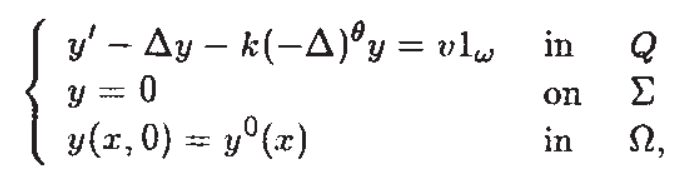

where $0 \leq \theta<1, k>0$ and $\Sigma=\Gamma \times(0, T), \Gamma=\partial \Omega$.

Both the state $y$ and the control $v$ depeud on the space-time variables $(x, t) \in Q$. In (1) $(-\Delta)^{\theta}$ denote the powers of the unbounded operator $-\Delta$ in $L^{2}(\Omega)$ with domain $D(-\Delta)=H^{2}(\Omega) \cap H_{0}^{1}(\Omega)$ (i.e. $-\Delta$ denotes the Dirichlet Laplacian). By ' we denote derivation with respect to time.

In (2.1), $1_{\omega}$ denotes the characteristic function of the open subset $\omega$ of $\Omega$. Therefore the control $v$ acts on the system through the subset $\omega$.

As the value of the parameter $k$ increases, system (2.1), in the absence of control (i.e. when $v=0$ ) becomes more and more unstable. This can be easily seen by computing the eigenvalues of $-\Delta+k(-\Delta)^{\theta}$ with Dirichlet boundary conditions. Indeed, if $0<\lambda_{1}<\lambda_{2} \leq \cdots \leq \lambda_{j} \leq \cdots$ are the eigenvalues of the Dirichlet Laplacian, the eigenvalues of this operator are $\lambda_{j}-k \lambda_{j}^{\theta}$. Therefore, as $k$ increases more and more eigenvalues become negative thus leading to a larger and larger unstable subspace of $L^{2}(\Omega)$ that when $k \nearrow \infty$, eventually, covers the whole $L^{2}(\Omega)$.

This paper is devoted to the analysis of how the controllability properties of system (2.1) behave as $k / \infty$.

We fix any $0 \leq \theta<1$ and consider the following three controllability problems: 


\section{Problem 1. Null-controllability.}

Given $y^{0} \in L^{2}(\Omega)$ we look for $v \in L^{2}(Q)$ such that the solution of (2.1) satisfies

$$
y(T)=0 \text { in } \Omega .
$$

The methods of [LR] and [LZ] allow us to show that for every $0 \leq \theta<1$ and $k>0$ system (2.1) is null-controllable. Therefore, for any $y^{0} \in$ $L^{2}(\Omega)$ the set of admissible controls

$$
\mathcal{U}_{a d}^{k}=\left\{v \in L^{2}(Q): y \text { solution of (2.1) satisfies (2.2) }\right\}
$$

is non-empty.

We introduce the quantity

$$
\mathcal{M}\left(k ; y^{0}, 0\right)=\inf _{v \in \mathcal{U}_{\mathrm{add}}^{k}} \frac{1}{2} \int_{0}^{T} \int_{\omega} v^{2} d x d t
$$

which measures the amount of control that is required to drive the initial state $y^{0}$ to zero.

This function gives now a precise definition in the present situation of $\phi\left(k ; y^{0}, 0\right)$ in (1.14). The quantity $\mathcal{M}$ depeuds on three parameters: $k$, which is the parameter in system (2.1) that measures its unstability; $y^{0}$, the initial data to be controlled and, on a third variable that takes account of the final condition to be reached and that, in this particular case, is always zero.

This problem will be studied in detail in section 3 . Roughly speaking we prove that

$$
\mathcal{M}\left(k ; y^{0}, 0\right) \rightarrow \infty \text { as } k \rightarrow \infty
$$

for any $y^{0} \not \equiv 0$. We also give some estimates on the growth of the function $\mathcal{M}\left(k ; y^{0}, 0\right)$.

This is a natural result. Indeed, as we mentioned above, as $k \rightarrow \infty$ the unstability of the equilibrium $y=0$ of system (2.5) increases and consequently, it becomes harder and harder to drive the solution to this equilibrium. 
The fact that the unstability of system (2.1) increases as $k$ increases suggests that it may become cheaper and cheaper to drive the initial data $y^{0}=0$ to a non-trivial final data $y^{1} \neq 0$. Indeed, in this case we are trying to quit the equilibrium $y^{0}=0$ whose unstability is increasing.

Therefore, let us consider now the case in which $y^{0}=0$. In other words, let us consider the system

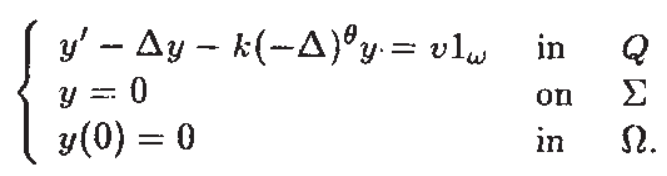

Due to the irreversibility of system (2.1) the exact controliability property does not hold and therefore the set of reachable states at time $T$ is strictly contained in $L^{2}(\Omega)$. It is then natural to relax the control requirement

$$
y(T)=y^{1} \quad \text { in } \quad \Omega
$$

at time $t=T$.

We consider here two different possibilities:

Problem 2. Approximate controllability from $y^{0}=0$.

Given any $y^{1} \in L^{2}(\Omega)$ and $\beta>0$ we relax condition (2.7) to

$$
\left\|y(T)-y^{1}\right\|_{L^{2}(\Omega)} \leq \beta
$$

or, in other words,

$$
y(T) \in y^{1}+\beta B
$$

where $B$ denotes the unit ball of $L^{2}(\Omega)$.

System (2.6) is approximately controllable and therefore the set of admissible controls

$$
\mathcal{U}_{a d}^{k}\left(y^{1} ; \beta\right)=\left\{v \in L^{2}(Q): y \text { solution of (2.6) satisfies (2.9) }\right\}
$$

is non-empty for any $\beta>0$ and $y^{1} \in L^{2}(\Omega)$.

We set

$$
\mathcal{M}\left(k ; 0, y^{\mathrm{k}}+\beta B\right)=\inf _{U_{a d}^{k}\left(y^{1} ; \beta\right)} \frac{1}{2} \int_{0}^{T} \int_{\omega} v^{2} d x d t .
$$


This function gives now a precise definition in the present situation of $\phi\left(k ; 0, \mathcal{M}\left(y^{1}\right)\right)$ of section 1 with $\mathcal{M}\left(y^{1}\right)=y^{1}+\beta B$.

The behavior of this function with respect to $k$ will be addressed in section 4. As we will see

$$
\mathcal{M}\left(k ; 0, y^{1}+\beta B\right) \rightarrow 0 \quad \text { as } \quad k \rightarrow \infty
$$

for any $y^{1} \in L^{2}(\Omega)$ and $\beta>0$ and this agrees with our intuition in the sense that more instability makes the control of the system cheaper. However we will only be able to prove (2.12) in two particular cases: (a) $\omega=\Omega$ and (b) $\theta=0$.

Problem 3. Finite-dimensional controllability from $y^{0}=0$.

Let $E$ be a finite-dimensional subspace of $L^{2}(\Omega)$ and let us denote by $\pi_{E}$ the orthogonal projection over $E$.

Given any $y^{1} \in L^{2}(\Omega)$ there exists some $v \in L^{2}(\Omega)$ such that the solution of (2.6) satisfies

$$
\pi_{E}\left(y(T)-y^{1}\right)=0 .
$$

This finite-dimensional controllability property is in fact an inmediate consequence of the approximate controllability property of Problem 2 above (see $[Z]$ ).

Therefore, the set

$$
\mathcal{U}_{a d}^{k}\left(0, \pi_{E}\left(y^{1}\right)\right)=\left\{v \in L^{2}(Q): y \text { solution of }(2.6) \text { satisfies }(2.13)\right\}
$$

is non-empty.

We set

$$
\mathcal{M}\left(k ; 0, \pi_{E}\left(y^{1}\right)\right)=\inf _{v \in \mathcal{U}_{a d}^{k}\left(0, \pi_{E}\left(y^{1}\right)\right)} \frac{1}{2} \int_{0}^{T} \int_{\omega} v^{2} d x d t
$$

This function gives now a precise definition of $\phi\left(k ; 0, \mathcal{M}\left(y^{1}\right)\right)$ with $\mathcal{M}\left(y^{1}\right)$ given as in (1.6). As we will see

$$
\mathcal{M}\left(k ; 0, \pi_{E}\left(y^{1}\right)\right) \rightarrow 0 \quad \text { as } \quad k \rightarrow \infty
$$

too. This will be done in section 5 in two cases: (a) when $E$ is fixed; (b) when $E$ is the span of the eigenfunctions of the laplacian associated to eigenvalues $\lambda \leq \mu(k)$ with $\mu(k)=k^{\delta}$ and some $\delta \leq 2$ depending on $\theta$. 
When considering any of the problems we have described it is useful to have in mind that all computations are rather explicit in the particular case in which $\theta=0$. Indeed, when $\theta=0$ the state equation reduces to

$$
y^{\prime}-\Delta y-k y=v 1_{\omega}
$$

This equation, by the change of variables $z=e^{-k t} y$ and $w=e^{-k t} v$ reduces to

$$
z^{\prime}-\Delta z=w 1_{\omega}
$$

Therefore, at this level, the dependence with respect to $k$ disappears. However, when dealing with Problems 2 and 3 one has to take into account that the final requirements at time $t=T$ change with this change of variables.

\section{The cost of controlling to zero}

\subsection{The main result}

Let us recall that $\mathcal{M}\left(k ; y^{0}, 0\right)$ measures the amount of control that is needed to drive the initial data $y^{0}$ to the zero equilibrium.

The main result of this section is as follows:

Theorem 3.1. For any $y^{0} \in L^{2}(\Omega), y^{0} \not \equiv 0$,

$$
\mathcal{M}\left(k ; y^{0}, 0\right) \rightarrow \infty \text { as } k \rightarrow \infty .
$$

More precisely, there exists $c>0$ (which depends on $y^{0}$ ) such that

$$
\mathcal{M}\left(k ; y^{0}, 0\right) \geq c k \text { as } k \rightarrow \infty .
$$

Remark 3.1. As we will see in Remarks 3.2 and 3.3 below the linear growth can be sharp or not, i.e. $\mathcal{M}\left(k ; y^{0}, 0\right)$ can grow exactly like $c k$ as $k \rightarrow \infty$ or, in other cases, it can grow faster than $c k$.

Remark 3.2. One could ask, according to the general question raised in the Introduction, what happens when $k \rightarrow-\infty$. We suspect that in that case $\mathcal{M} \rightarrow 0$. But this is an open question. One can go further if one 
weakens the notion of controlling to zero. This will be presented in Section 3.3 below.

\section{Proof of Theorem 3.1.}

Let us consider the adjoint system

$$
\left\{\begin{array}{ccc}
-\varphi^{\prime}-\Delta \varphi-k(-\Delta)^{\theta} \varphi=0 & \text { in } & Q \\
\varphi=0 & \text { on } & \Sigma \\
\varphi(T)=\varphi^{0} & \text { in } & \Omega
\end{array}\right.
$$

The null controllability of system (2.1) is equivalent to the existence of a positive constant $C_{k}>0$ such that the following holds:

$$
\|\varphi(0)\|_{L^{2}(\Omega)}^{2} \leq C_{k} \int_{0}^{T} \int_{\omega} \varphi^{2} d x d t, \forall \varphi^{0} \in L^{2}(\Omega) .
$$

More precisely, there exists $C_{k}>0$ such that (3.4) holds if and only if for every $y^{0} \in L^{2}(\Omega)$ there exists $v \in L^{2}(Q)$ such that the solution of (2.1) satisfies

$$
y(T)=0 \text { in } \Omega
$$

and the mapping $y^{0} \in L^{2}(\Omega) \rightarrow v \in L^{2}(Q)$ is continuous.

The methods of [LR] and [LZ] allow to prove that system (2.1) is null-controllable and therefore there exists a constant $C_{k}$ such that (3.4) holds.

By duality (see J.-L. Lions [Li], vol. 2) one can show that the control $v_{k}$ of minimal norm such that

$$
\frac{1}{2} \int_{0}^{T} \int_{\omega} v_{k}^{2} d x d t=\mathcal{M}\left(k ; y^{0}, 0\right)=\inf _{v \in \mathcal{U}_{a d}^{k}} \frac{1}{2} \int_{0}^{T} \int_{\omega} v^{2} d x d t
$$

(according to the notations of $(2.3)-(2.4)$ ) is given by

$$
\left\{\begin{array}{l}
v_{k}=\varphi \text { in } \omega \times(0, T) \text { with } \varphi \text { solution of }(3.2) \text { where } \\
\varphi^{0} \text { minimizes } I_{k}\left(\cdot ; y^{0}\right) \text { below in the Hilbert } \\
\text { space of data } \varphi^{0} \text { such that } \varphi(0) \in L^{2}(\Omega)
\end{array}\right.
$$

where

$$
J_{k}\left(\varphi^{0} ; y^{0}\right)=\frac{1}{2} \int_{0}^{T} \int_{\omega} \varphi^{2} d x d t+\int_{\Omega} \varphi(0) y^{0} d x
$$


Once (3.4) holds it is easy to see that $J_{k}\left(\cdot ; y^{0}\right)$ has an unique minimizer such that $\varphi(0) \in L^{2}(\Omega)$ and therefore the optimal control $v_{k}$ is uniquenely determined by (3.6).

On the other hand

$$
\frac{1}{2} \int_{0}^{T} \int_{\omega} v_{k}^{2} d x d t=\mathcal{M}\left(k ; y^{0}, 0\right)=-\min _{\left\{\varphi^{0}: \varphi(0) \in L^{2}(\Omega)\right\}} J_{k}\left(\varphi^{0} ; y^{0}\right)
$$

Therefore (3.1) is equivalent to prove that

$$
\min _{\left\{\varphi: \varphi(0) \in L^{2}(\Omega)\right\}} J_{k}\left(\varphi^{0} ; y^{0}\right) \rightarrow-\infty \text { as } k \rightarrow \infty .
$$

To show (3.9) we evaluate the functional $J_{k}$ over particular choices of the initial data $\varphi^{0}$.

To do that we denote by $0<\lambda_{1}<\lambda_{2} \leq \cdots \leq \lambda_{j} \leq \cdots \rightarrow \infty$ the eigenvalues of $-\Delta$ in $H_{0}^{1}(\Omega)$ and by $\left\{w_{j}\right\}$ the corresponding sequence of eigenfunctions constituting an orthonormal basis of $L^{2}(\Omega)$.

We choose $\varphi^{0}=\rho w_{j}$ with $\rho \in \mathbb{R}$ and $j \in \mathbb{N}$. Then the solution $\varphi$ of (3.2) can be computed explicitely in separated variables:

$$
\varphi=\rho e^{\left(k \lambda \lambda_{j}^{\theta}-\lambda_{j}\right)(T-t)} w_{j}(x) .
$$

Then

$$
J_{k}\left(\varphi^{0}\right)=J\left(\rho w_{j}\right)=\frac{a_{j}}{2} \rho^{2}+b_{j} \rho
$$

where

$$
a_{j}=\left(\frac{e^{2\left(k \lambda_{j}^{\theta}-\lambda_{j}\right) T}-1}{2\left(k \lambda_{j}^{\theta}-\lambda_{j}\right)}\right) \int_{\omega} w_{j}^{2} d x ; b_{j}=e^{\left(k \lambda_{j}^{\theta}-\lambda_{j}\right) T} \int_{\Omega} y^{0} w_{j} d x .
$$

Observe that in (3.11) and in the sequel we drop $y^{0}$ from the variables on which $J_{k}$ depends to simplify the notation.

Then, for any $j \in \mathbb{N}$,

$$
\inf _{\rho \in \mathbb{R}} J_{k}\left(\rho w_{j}\right)=-\frac{b_{j}^{2}}{2 a_{j}}
$$

and obviously

$$
\min _{\left\{\varphi^{0}: \varphi(0) \in L^{2}(\Omega)\right\}} J_{k}\left(\varphi^{0}\right) \leq \inf _{\rho \in \mathbb{R}} J_{k}\left(\rho w_{j}\right)=-\frac{b_{j}^{2}}{2 a_{j}} .
$$


Let us analyze now the quantity $b_{j}^{2} / 2 a_{j}$ :

$$
\frac{b_{j}^{2}}{2 a_{j}}=\frac{e^{2\left(k \lambda_{j}^{\theta}-\lambda_{j}\right) T}\left(k \lambda_{j}^{\theta}-\lambda_{j}\right)\left(\int_{\AA} y^{0} w_{j} d x\right)^{2}}{\left(e^{2\left(k \lambda_{j}^{\theta}-\lambda_{j}\right) T}-1\right) \int_{\omega} w_{j}^{2} d x} .
$$

It is then olear that, for $j \in \mathbb{N}$ fixed,

$$
\frac{b_{j}^{2}}{2 a_{j}} \sim k \frac{\lambda_{j}^{\theta}\left(\int_{\Omega} y^{0} w_{j} d x\right)^{2}}{\int_{\omega} w_{j}^{2} d x} .
$$

In particular

$$
\lim _{\rho \rightarrow \infty} \frac{b_{j}^{2}}{2 a_{j}}=\infty, \forall j \in \mathbb{N}: \int_{\Omega} y^{0} w_{j} d x \neq 0
$$

and in view of (3.8)-(3.14) this concludes the proof of the Theorem.

Remark 3.3. According to the proof of the theorem, (3.2) holds with

$$
c=\sup _{j \in \mathbb{N}} \frac{\lambda_{j}^{\theta}\left(\int_{\Omega} y^{0} w_{j}\right)^{2}}{\int_{\omega} w_{j}^{2}}
$$

It is easy to see that in some particular cases this constant diverges. Indeed, let us consider the one-dimensional case in which $\Omega=(0,1)$ and $\omega=\Omega$, i.e. the control acts everywhere in $\Omega$. Then $\lambda_{j}=(j \pi)^{2}$. On the other hand, there exists $y^{0} \in L^{2}(\Omega)$ such that $\left(\int_{\Omega} y^{0} w_{j}\right)^{2} \sim j^{-1-\delta}$ as $j \rightarrow \infty$ for any $\delta>0$.

Going back to formula (3.18) we see that

$$
\frac{\lambda_{j}^{\theta}\left(\int_{\Omega} y^{0} w_{j}\right)^{2}}{\int_{\omega} w_{j}^{2}} \sim j^{2(\theta-1 / 2)-\delta} .
$$


Therefore, as soon as $\theta>1 / 2$, if a is defined as in (3.18), then $c=\infty$ for suitable choices of $y^{0} \in L^{2}(\Omega)$. This shows that the linear growth estimate of (3.2) for $\mathcal{M}(k)$ with respect to $k$ is not optimal in general. We will obtain sharper estimates in section 3.2 below.

Remark 3.4. It is not hard to see that the linear growth estimate (2.2) is sharp in some cases.

Consider the case in which $\omega=\Omega$ and $y^{0}=w_{j_{0}}$, for some $j_{0} \in \mathbb{N}$, i.e. $y^{0}$ is chosen to be an eigenfunction of the Dirichlet Laplacian.

Then, if $\varphi^{0}=\sum_{j \geq 1} \alpha_{j} w_{j}$; we have

$$
J_{k}\left(\varphi^{0} ; w_{j_{0}}\right)=\frac{1}{2} \sum_{j \geq 1} \alpha_{j}^{2}\left(\frac{e^{2\left(k \lambda_{j}^{\theta}-\lambda_{j}\right) T}-1}{2\left(k \lambda_{j}^{\theta}-\lambda_{j}\right)-}\right)+e^{\left(k \lambda_{j_{0}}^{\theta}-\lambda_{j_{0}}\right) T} \alpha_{j_{0}}
$$

It is clear that the minimun of $J_{k}$ is achieved on the one-dimensional subspace generated by $w_{j_{0}}$. Proceeding as in the proof of Theorem 3.1 we deduce that

$$
\mathcal{M}\left(k ; w_{j_{0}}, 0\right)=\frac{e^{2\left(k \lambda_{j_{0}}^{\theta}-\lambda_{j_{0}}\right) T}\left(k \lambda_{j_{0}}^{\theta}-\lambda_{j_{0}}\right)}{\left(e^{2\left(k \lambda_{j_{0}}^{\theta}-\lambda_{j_{0}}\right) T}-1\right)} \sim \lambda_{j_{0}}^{\theta} k \text { as } k \rightarrow \infty .
$$

\subsection{Further estimates.}

The object of this section is to show that, on particular examples, the linear growth estimate of (3.2) can be improved in the sense that $\mathcal{M}$ grows faster.

We consider some particular cases:

\section{Example 1. One space dimension.}

We set $\Omega=(0,1)$ so that $w_{j}(x)=\sqrt{2} \sin (j \pi x)$ and $\lambda_{j}=(j \pi)^{2}$. We choose $\omega=(\alpha, \beta)$ with $0 \leq \alpha<\beta \leq 1$. 
According to (3.8)-(3.14), given any $y^{0} \in L^{2}(0,1)$ we have

$$
\mathcal{M}\left(k ; y^{0}, 0\right) \geq \frac{e^{2\left(k(j \pi)^{2 \theta}-(j \pi)^{2}\right) T}\left(k(j \pi)^{2 \theta}-(j \pi)^{2}\right)\left(\int_{0}^{1} y^{0} \sin (j \pi x)\right)^{2}}{\left(e^{2\left(k(j \pi)^{2 \theta}-(j \pi)^{2}\right) T}-1\right) \int_{\alpha}^{\beta} \sin ^{2}(j \pi x)} .
$$

We choose $j$ so that $f_{k}(j)=k(j \pi)^{2 \theta}-(j \pi)^{2}$ is maximum. Equivalently,

$$
(\pi j)^{2}=(k \theta)^{\frac{1}{1-\theta}} \Leftrightarrow j=\frac{1}{\pi}(k \theta)^{\frac{1}{2(1-\theta)}} .
$$

However, this choice of $k$ does not guarantee that $j \in \mathbb{N}$. Thus, let us consider the sequence $k_{j} \rightarrow \infty$ such that all the $j$-s given by (3.20) belong to $N$.

The value of $f_{k}(j)$ at this point is $\left(\theta^{\theta / 1-\theta}-\theta^{1 / 1-\theta}\right) k^{1 / 1-\theta}=c_{\theta} k^{1 / 1-\theta}$ with $c_{\theta}=\theta^{\theta / 1-\theta}-\theta^{1 / 1-\theta}>0$. Thus, with this choice of $k=k_{j}$ :

$$
\mathcal{M}\left(k ; y^{0}, 0\right) \geq \frac{e^{2 c_{\theta} T k^{1 /(1-\theta)}} e^{2 c_{\theta} k^{1 /(1-\theta) T}}}{\left(\int_{\alpha}^{1 /(1-\theta)} \sin (j \pi x)\right)^{2}}
$$

Since $\int_{\alpha}^{\beta} \sin ^{2}(j \pi x) d x$ remains bounded above and below by positive constants we see that there exists $\gamma_{\theta}>0$ such that

$$
\mathcal{M}\left(k ; y^{0}, 0\right) \geq \gamma_{\theta} k^{1 /(1-\theta)}\left(\int_{0}^{1} y^{0} \sin (j \pi x)\right)^{2} .
$$

In view of (3.21) it is clear that the rate of growth of $\mathcal{M}(k)$ depends on the initial datum $y^{0}$ under consideration. For instance, if

$$
\int_{\Omega} y^{0} \sin (j \pi x) \sim c j^{-\frac{1}{2}-\delta}, j \rightarrow \infty
$$

for some $c>0$ and $\delta>0$ (observe that this guarantees $\sum_{j=1}^{\infty}\left(\int_{\Omega} y^{0} \sin (j \pi x) d x\right)^{2}<\infty$ or, in other words, $\left.y^{0} \in L^{2}(0,1)\right)$, taking into account that $j$ is of the order of $k^{1 / 2(1-\theta)}$ we deduce that

$$
\mathcal{M}\left(k ; y^{0}, 0\right) \geq \gamma_{\theta} k^{(1 /(1-\theta)-1 / 2(1-\theta)-\delta /(1-\theta))}=\gamma_{\theta} k^{1 / 2(1-\theta)-\delta /(1-\theta)} .
$$


Therefore, for suitable choices of the initial data we see that the estimate (3.2) may be replaced by (3.23) along the subsequence $k_{j}$ such that (3.20) holds.

Observe that $1>1 / 2(1-\theta)$ if and only if $\theta<1 / 2$. Therefore (3.23) only shows a faster growth than (3.2) in the range $\theta>1 / 2$.

Let us also check that (3.23) holds not only for the sequence $k_{j}$ but for the whole family $k \rightarrow \infty$. Indeed, for any $k>0$ sufficiently large we choose $j=\left[\frac{1}{\pi}(k \theta)^{1 / 2(1-\theta)}\right]$ where $H$ denotes the integer part. Then

$$
f\left(\left[\frac{1}{\pi}(k \cdot \theta)^{1 / 2(1-\theta)}\right]\right)=f\left(\frac{1}{\pi}(k \theta)^{1 / 2(1-\theta)}\right)+\varepsilon=c_{\theta} k^{1 /(1-\theta)}+\varepsilon
$$

with

$$
|\varepsilon| \leq\left|f^{\prime}\left(\frac{1}{\pi}(k \theta)^{1 / 2(1-\theta)}\right)\right| \leq C\left[k^{1+\frac{2 \theta-1}{2(1-\theta)}}+k^{\frac{1}{2(1-\theta)}}\right]=O\left(k^{\frac{1}{2(1-\theta)}}\right) .
$$

Therefore

$$
f\left(\left[\frac{1}{\pi}(k \theta)^{1 / 2(1-\theta)}\right]\right) \sim k^{1 /(1-\theta)} \text { as } k \rightarrow \infty .
$$

We conclude that (3.23) holds without extracting a subsequence.

Therefore we have proved that:

Proposition 3.1. In one space dimension and when $\theta>1 / 2$, for every $1<\gamma<1 / 2(1-\theta)$ there exists $y^{0} \in L^{2}(\Omega)$ such that

$$
\mathcal{M}\left(k ; y^{0}, 0\right) \geq c k^{\gamma} \text { as } k \rightarrow \infty
$$

for some $\mathrm{c}>0$.

Example 2. Several space dimensions and $\omega=$ neighborhood of the boundary.

Let us consider now the general case of a bounded surooth domain $\Omega$ of $\mathbb{R}^{n}, n \geq 1$. Suppose that the support of the control $\omega$ is a neighborhood of $\partial \Omega$ in $\Omega$. In other words, $\omega=\mathcal{O} \cap \Omega$ where $\mathcal{O}$ is a neighborhood of $\partial \Omega$ in $\mathbb{R}^{n}$.

In this geometrical setting it is well-known that when the control time is large enough the wave equation is exactly controllable in $H_{0}^{1}(\Omega) \times$ $L^{2}(\Omega)$ with $L^{2}$-controls supported in $\omega$ (see [Li1], vol. 1). 
As a consequence of this we deduce easily the existence of a positive constant $c>0$ such that

$$
0<c \leq \int_{\omega} w_{j}^{2} d x, \forall j \in \mathbb{N} .
$$

Of course, condition (3.24) holds for more general subdomains $\omega$. For instance, from [Li1, vol. 1] we know that it holds when $\omega$ is a neighborhood of a subset of $\partial \Omega$ of the form $\Gamma\left(x^{0}\right)=\left\{x \in \partial \Omega:\left(x-x^{0}\right) \cdot \nu(x)>0\right\}$ for some $x^{0} \in \mathbb{R}^{n}(\nu(x)$ denotes the outward unit normal to $\Omega$ at $w \in \partial \Omega$ and - the scalar product in $\left.\mathbb{R}^{n}\right)$. More general sufficient conditions for (3.24) to hold may be obtained in terms of the propagation of bicharacteristic rays (see [BLR] and Appendix II in [Lil, vol. 1]).

We reproduce the computations of Example 1 above.

According to (3.8) and (3.14) we have

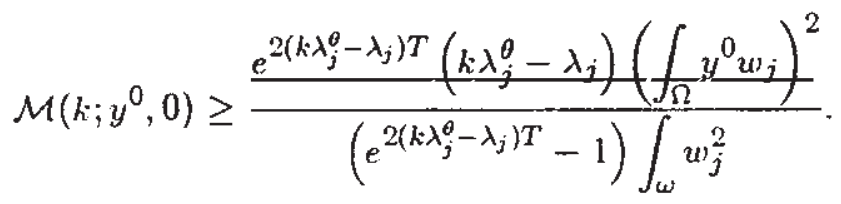

Recall that the maximum value of the function $f_{k}(\lambda)=k \lambda^{\theta}-\lambda$ is achieved when

$$
\lambda=(\theta k)^{1 /(1-\theta)} .
$$

It is clear that (3.26) provides an eigenvalue of $-\Delta$ in $H_{0}^{1}(\Omega)$ only for suitable values of $k$. We choose the subsequence $j=\ell^{\sigma}, \ell \in \mathbb{N}$ with $\sigma>0$ to be fixed later on. We set

$$
k_{\ell}=\frac{1}{\theta}\left(\lambda_{\ell^{\sigma}}\right)^{1-\theta} \text {. }
$$

We analyze the lower bound on the right hand side of (3.25) as $(\rightarrow \infty$ by taking $j=\ell^{\sigma}$ and $k$ as in (3.27).

Since $\max _{\lambda>0} f_{k}(\lambda)=c_{\theta} k^{1 /(1-\theta)}$, in view of (3.24) we get

$$
\mathcal{M}\left(k_{\ell} ; y^{0}, 0\right) \geq C h_{\ell}^{1 /(1-\theta)}\left(\int_{\Omega} y^{0} u_{\ell^{\sigma}}\right)^{2} .
$$

We now recall the existence of a constant $c>0$ such that $\lambda_{j} \geq c j^{2 / n}$, $n$ being the space dimension. Then

$$
\lambda_{j}=\lambda_{\ell \sigma} \geq c \ell^{2 \sigma / n} \text {. }
$$


We choose the initial data $y^{0} \in L^{2}(\Omega)$ such that its Fourier coefficients $\int_{\Omega} y^{0} w_{j}$ are non zero if and only if $j=\ell^{\sigma}$ for some $\ell \in \mathbb{N}$. Then, according to (3.29) we can choose $y^{0} \in L^{2}(\Omega)$ such that

$$
\left(\int_{\Omega} y^{0} w_{\ell} \sigma d x\right)^{2} \sim \lambda_{\ell \sigma}^{-\frac{n}{2 \sigma}-\delta}, \text { as } \ell \rightarrow \infty
$$

for some $\delta>0$.

In view of $(3.27),(3.28)$ and $(3.30)$ we deduce

$$
\mathcal{M}\left(k_{\ell} ; y^{0}, 0\right) \geq c k_{\ell}^{\frac{1}{1-\theta}\left(1-\frac{\pi}{2 \sigma}\right)-\frac{b}{1-\theta}} .
$$

Observe that $(1-n / 2 \sigma) /(1-\theta)>1$ if and only if $\theta>\frac{n}{2 \sigma}$ which holds for any $\theta>0$ provided $\sigma$ is chosen sufficiently large (i.e. $\sigma>n / 2 \theta$ ).

We have proved the following result:

Proposition 3.2. Let $\Omega$ be any bounded smooth domain of $\mathbb{R}^{n}$ and $\omega$ a neighborhood of its boundary. Then, for any $\theta>0$ and $1<\gamma<1 /(1-\theta)$ there exists $y^{0} \in L^{2}(\Omega)$ such that

$$
\limsup _{k \rightarrow \infty} \frac{\mathcal{M}\left(k ; y^{0}, 0\right)}{k^{\gamma}}>0
$$

Remark 3.5. The result stated in Proposition 3.2 is much weaker than the one we have proved in Proposition 3.1 for space dimension $n=1$.

Indeed, while in one space dimension

$$
\mathcal{M}\left(k ; y^{0}, 0\right) \geq c k^{\gamma}
$$

holds for all $k \rightarrow \infty$, in Proposition 3.2 we find a subsequence $k_{\ell} \rightarrow$ $\infty$ such that this inequality holds. Observe that $k_{\ell}$ is of the order of $k_{\ell} \sim \ell^{\frac{2 \sigma(1-\theta)}{n}}$ with $\sigma>n / 2 \theta$ and therefore $k_{\ell}>>\ell^{(1-\theta) / \theta}$. Thus the subsequence $k_{\ell}$ grows faster and faster as $\theta \rightarrow 0$

\section{Example 3. Several space dimensions and exceptional $\omega$.}

In Example 2 above we have considered the case in which (3.24) holds. However, it is well known that for some particular domains $\Omega$ 
there exist subdomains $\omega$ for which there exists a subsequence of eigenfunctions $\left\{w_{j(\ell)}\right\}$ such that

$$
\int_{\omega} w_{j(\ell)}^{2} d x \longrightarrow 0 \text { as } \ell \rightarrow \infty
$$

This is for instance the case when $\Omega$ is the unit ball and $\omega$ is the ball of radious $r$ with $r<1$. Indeed, in this case it is well known that there exist eigenfunctions whose energy is concentrated on a neighborhood of the boundary and for which (3.33) holds (see for instance [CMZ]).

For these subsequences of eigenfunctions, since $\int_{\omega} w_{j}^{2} d x$ appears in the denominator, the rate of growth may be faster than in (3.32).

Example 4. $\theta=0$.

When $\theta=0$ all the computations can be done explicitely. Indeed, in this particular case, system (2.1) reads as follows

$$
\left\{\begin{array}{lll}
y^{\prime}-\Delta y-k y=v 1_{\omega} & \text { in } & Q \\
y=0 & \text { on } & \Sigma \\
y(0)=y^{0} & \text { in } & \Omega .
\end{array}\right.
$$

Observe that if $v$ is such that the solution $y$ of (3.34) satisfies $y(T)=$ 0 . Then $z=y e^{-k t}$ is such that

$$
\left\{\begin{array}{lll}
z^{\prime}-\Delta z=p 1_{\omega} & \text { in } & Q \\
z=0 & \text { on } & \Sigma \\
z(0)=y^{0} & \text { in } & \Omega
\end{array}\right.
$$

with $p=e^{-k t} v$ and $z\left(T^{\prime}\right)=0$.

Let us see that

$$
\mathcal{M}\left(k ; y^{0}, 0\right) \rightarrow \infty \text { as } k \rightarrow \infty
$$

as soon as $y^{0} \not \neq 0$.

We argue by contradiction. If (3.36) does not hold there exists a sequence $k_{j} \rightarrow \infty$ and controls $v_{j}$ such that the solution $y_{k_{j}}$ of $(3.34)$ satisfy $y_{k_{j}}(T)=0$ and

$$
\int_{0}^{T} \int_{\omega}\left|v_{k_{j}}\right|^{2} \leq C<\infty, \forall_{j}
$$


According to the change of variables above this implies the existence of a sequence of controls $p_{k_{j}}=e^{-k_{j} t} v_{k_{j}}$ for (3.35) such that the corresponding solution $z_{k_{j}}$ satisfies

$$
z_{k_{j}}(T)=0
$$

and moreover, in view of (3.37),

$$
\int_{0}^{T} \int_{\omega} e^{2 k_{j} t}\left|p_{k_{j}}\right|^{2} d x d t \leq C<\infty, \forall j
$$

From (3.39) we deduce that $p_{k_{f}}$ is bounded in $L^{2}(\omega \times(0, T))$ and moreover

$$
p_{k_{j}} \rightarrow 0 \text { in } L_{l o c}^{2}\left(0, T ; L^{2}(\omega)\right)
$$

and

$$
p_{k_{j}} \rightarrow 0 \text { weakly in } L^{2}(\omega \times(0, T)) .
$$

By the regularizing effect of the heat equation, from (3.41) we deduce that

$$
z_{k_{j}}(T) \rightarrow z(T) \text { in } L^{2}(\Omega)
$$

where $z$ solves

$$
\left\{\begin{array}{lll}
z^{\prime}-\Delta z=0 & \text { in } & Q \\
z=0 & \text { on } & \Sigma \\
z(0)=y^{0} & \text { in } & \Omega .
\end{array}\right.
$$

Clearly (3.38) and (3.42) are in contradiction since $z(T) \not \equiv 0$ whenever $y^{0} \not \equiv 0$.

\subsection{Weakening of the notion of controlling to zero.}

Let us introduce

$$
\left\{\begin{array}{l}
E_{\mu}=\operatorname{span}\left\{w_{j}: \lambda_{j} \leq \mu\right\} \text { and } \pi_{\mu}=\text { orthogonal } \\
\text { projection from } L^{2}(\Omega) \text { onto } E_{\mu} .
\end{array}\right.
$$

We then define

$$
\mathcal{M}_{\mu}\left(k ; y^{0}, 0\right)=\inf _{v} \frac{1}{2} \int_{0}^{T} \int_{\omega} v^{2} d x d t
$$

where $v$ is such that

$$
\pi_{\mu} y(T ; v)=0
$$


If we denote by $\mathcal{U}_{a d, \mu}^{k}$ the set of $v^{\prime} s$ such that (3.46) holds true, we have obviously

$$
\mathcal{U}_{a d, \mu}^{k} \supset \mathcal{U}_{a d}^{k} \text { (as defined in (2.3)) }
$$

so that

$$
\mathcal{M}_{\mu}\left(k ; y^{0}, 0\right) \leq \mathcal{M}\left(k ; y^{0}, 0\right) .
$$

Using duality as in Section 3.1 above, we have

$$
\mathcal{M}_{\mu}\left(k ; y^{0}, 0\right)=-\inf _{\varphi^{0} \in E_{\mu}} J_{k}\left(\varphi^{0}, y^{0}\right)
$$

(compare to $(3.8)$ where $\varphi^{0}$ spans an infinite-dimensional subspace of $\left.L^{2}(\Omega)\right)$.

The arguments of section 3.1 apply provided one can find $w_{j} \in E_{\mu}$ such that

$$
\left(y^{0}, w_{j}\right) \neq 0
$$

i.e.

$$
y^{0} \notin E_{\mu}^{1}
$$

(a condition which is always satisfied if $y^{0} \neq 0$ when $\mu=+\infty, E_{\mu}=$ $\left.L^{2}(\Omega)\right)$.

Therefore,

Theorem 3.2. Provided $y^{0}$ satisfies (3.50), one has

$$
\mathcal{M}_{\mu}\left(k ; y^{0}, 0\right) \rightarrow+\infty \text { as } k \rightarrow+\infty \text {. }
$$

One can go one step further in that case:

Theorem 3.3. We assume that (3.50) holds true. Then there exist two positive constants $\gamma_{1}, \gamma_{2}$ such that

$$
\gamma_{1} k \leq \mathcal{M}_{\mu}\left(k ; y^{0}, 0\right) \leq \gamma_{2} k
$$

as $k \rightarrow+\infty$.

\section{Proof of Theorem 3.3.}

The lower bound in (3.52) can be proved as in the proof of Theorem 3.1. 
To prove the upper bound, let us take

$$
\varphi^{0}=\sum_{\lambda_{j} \leq \mu} f_{j} w_{j}
$$

Then

$$
\varphi=\sum_{\lambda_{j} \leq \mu} \varphi_{j}(l) f_{j} w_{j}
$$

where

$$
-\varphi_{j}^{\prime}+\left(\lambda_{j}-k \lambda_{j}^{\theta}\right) \varphi_{j}=0, \varphi_{j}(T)=1
$$

and

$$
J_{k}\left(\varphi^{0} ; y^{0}\right)=\frac{1}{2} \int_{0}^{T} d t \int_{\omega}\left(\sum_{\lambda_{j} \leq \mu} \varphi_{j}(t) f_{j} w_{j}\right)^{2} d x+\sum_{\lambda_{j} \leq \mu} \varphi_{j}(0) \int_{\Omega} y^{0} w_{j} d x
$$

Let us recall now the following result from [LR] and [LZ]:

Lemma 3.1. Assume that $\Omega$ is a bounded domain of $\mathbb{R}^{n}$ of class $C^{\infty}$. Let $\omega$ be any open non-empty subset of $\Omega$. Then, there exist two constants $C_{1}, C_{2}>0$ such that

$$
\int_{\omega}\left|\sum_{\lambda_{j} \leq \mu} a_{j} w_{j}(x)\right|^{2} d x \geq C_{1} e^{-C_{2} \sqrt{\mu}} \sum_{\lambda_{j} \leq \mu}\left|a_{j}\right|^{2}
$$

for every $\mu$ and every $\left\{a_{j}\right\} \in \mathfrak{l}^{2}$.

Then

$$
\inf J_{k}\left(\psi^{0}, y^{0}\right) \geq \sum_{\lambda_{j} \leq \mu} \inf _{f_{j} \in \mathbb{R}}\left[\frac{c}{2} \alpha_{j} f_{j}^{2}+\beta_{j} f_{j}\right]
$$

where

$$
c=c_{1} e^{-c_{2} \sqrt{\mu}}, \alpha_{j}=\int_{0}^{T} \varphi_{j}^{2}(t) d t, \beta_{j}=\varphi_{j}(0) \int_{\Omega} y^{0} w_{j} d x
$$

i.e.

$$
\inf J_{k}\left(\varphi^{0}, y^{0}\right) \geq-\sum_{\lambda_{j} \leq \mu} \frac{\beta_{j}^{2}}{2 c \alpha, j}
$$


or

$$
\mathcal{M}_{\mu}\left(k ; y^{0}, 0\right) \leq \sum_{\lambda_{j} \leq \mu} \frac{\beta_{j}^{2}}{2 c \alpha_{j}} .
$$

But $\varphi_{j}=e^{-\left(\lambda_{j}-k \lambda_{j} \theta\right)}(T-t)$ so that $(3.60)$ can be written in an explicit form

$$
\mathcal{M}_{\mu}\left(k ; y^{0}, 0\right) \leq \frac{e^{c_{2} \sqrt{\mu}}}{c_{1}} \sum_{\lambda_{j} \leq \mu} \frac{e^{2\left(k \lambda_{j}^{\theta}-\lambda_{j}\right) T}\left(\int_{\Omega} y^{0} u_{j} d x\right)^{2}\left(k \lambda_{j}^{\theta}-\lambda_{j}\right)}{e^{2\left(k \lambda_{j}^{\theta}-\lambda_{j}\right) T}-1}
$$

hence the result follows.

Remark 3.6. Note that Lemma 3.1 is only required to make explicit. the dependence of the upper bound (3.61) with respect to $\mu$. Otherwise it is sufficient to use the fact that $\left\{w_{j}\right\}_{\lambda_{j} \leq \mu}$ are linearly independent in $L^{2}(\omega)$.

Remark 3.7. It is interesting to notice that (3.61) (which is valid for $k \rightarrow-\infty$ ) implies

Theorem 3.4. When $k \rightarrow-\infty$ one has

$$
\mathcal{M}_{\mu}\left(k ; y^{0}, 0\right) \rightarrow 0 \text { exponentially fast. }
$$

We notice that this result follows the botton line of this paper. It becomes cheaper and cheaper to control to zero systems which are more and more stable but we emphasize that this is proven only for $\mathcal{M}_{\mu}\left(k ; y^{0}, 0\right)$ with $\mu$ finite.

\section{Controlling to a ball from $y^{0}=0$}

We consider now the system with null initial data

$$
\left\{\begin{array}{lll}
y^{\prime}-\Delta y-k(-\Delta)^{\theta} y=v 1_{\omega} & \text { in } & Q \\
y=0 & \text { on } & \Sigma \\
y(0)=0 & \text { in } & \Omega
\end{array}\right.
$$


Given $y^{1} \in L^{2}(\Omega)$ and $\beta>0$ we look for $v \in L^{2}(Q)$ such that

$$
y(T) \in y^{1}+\beta B .
$$

The fact that this approximate controllability problem is solvable for every $y^{1} \in L^{2}(\Omega)$ and $\beta>0$ is a consequence of the following unique continuation property for the dual system: If $\varphi$ solves

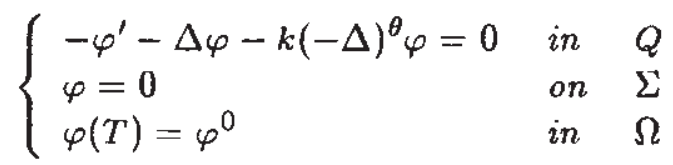

and $\varphi=0$ in $\omega \times(0, T)$, then $\varphi^{0} \equiv 0$.

This uniqueness property can not be derived from Holmgrèn's Uniqueness Theorem since the equation in (4.3) is an integro-differential equation. However using the analyticity of the semigroup generated by (4.3) the uniqueness problem can be reduced to the case where $\varphi=$ $\alpha e^{-\left(\lambda_{j}-k \lambda_{j}^{\theta}\right)(T-t)} w_{j}(x)$ for some $\alpha \in \mathbb{R}$ and $j \in \mathbb{N}$. In this case the uniqueness holds trivially since $w_{j}$ are the eigenfunctions of $-\Delta$ in $H_{0}^{1}(\Omega)$ and therefore $w_{j}=0$ in $\omega$ implies immediately that $w_{j} \equiv 0$. We refer to the Appendix at the end of the paper for the details of the proof and to $[\mathrm{LiZ1}, 2]$ for various applications of this kind of arguments.

This shows that the set of admissible controls

$$
\mathcal{U}_{a d}^{k}\left(y^{1} ; \beta\right)=\left\{v \in L^{2}(Q): y \text { solution of (3.1) satisfies (3.2) }\right\}
$$

is non empty.

We define the cost of controlling to $y^{1}+\beta B$ as

$$
\mathcal{M}\left(k ; 0, y^{1}+\beta B\right)=\inf _{v \in \mathcal{U}_{a d}^{k}\left(y^{1}, \beta\right)} \frac{1}{2} \int_{0}^{T} \int_{\omega} v^{2} d x d t
$$

The optimal control $v$ that realizes the infimum in (4.5) may be characterized as follows:

$$
\left\{\begin{array}{l}
v=\varphi \text { where } \varphi \text { solves }(4.3) \text { with } \\
\varphi^{\theta} \text { the minimizer of } J_{k}\left(\varphi^{0} ; y^{1}, \beta\right) \text { below in } L^{2}(\Omega)
\end{array}\right.
$$

with

$$
J_{k}\left(\varphi^{0} ; y^{1}, \beta\right)=\frac{1}{2} \int_{0}^{T} \int_{\omega} \varphi^{2} d x d t+\beta\left\|\varphi^{0}\right\|_{L^{2}(\Omega)}-\int_{\Omega} y^{1} \varphi^{0} d x .
$$


We refer to [Li2] and [FPZ] for similar questions in the context of the classical heat equation.

Taking into account that the unstable subspace of system (4.1) increases as $k$ increases it seems natural to expect that

$$
\mathcal{M}\left(k ; 0, y^{1}+\beta B\right) \rightarrow 0 \text { as } k \rightarrow \infty .
$$

We are going to prove that this does indeed hold in two particular cases. The general case remains open and it is unclear whether this property will still be true when $\omega \subset \Omega, \omega \neq \Omega$ and $\theta>0$.

Example 1: $\omega=\Omega$.

In this case the control $v$ acts everywhere in $\Omega$. We can try to reproduce the arguments of Example 4 in section 3 above.

Let us consider first the heat equation

$$
\left\{\begin{array}{lll}
z^{\prime}-\Delta z=p & \text { in } & Q \\
z=0 & \text { on } & \Sigma \\
z(0)=0 . & &
\end{array}\right.
$$

Given $y^{1}$ and $\beta$ let $p$ be the optimal control (the one of minimal $L^{2}-$ norm) for (4.8) such that

$$
z(T) \in y^{1}+\beta B
$$

The control $p$ may be developed in Fourier series

$$
p=\sum_{j \geq 1} p_{j}(t) w_{j}(x)
$$

and then

$$
z=\sum_{j \geq 1} \int_{0}^{t} e^{-\lambda_{j}(t-s)} p_{j}(s) d s w_{j}(x) .
$$

We now look for a control $v$ such that the solution of (4.1) satisfies

$$
y(T)=z(T)
$$

and therefore, in particular, (4.2) holds.

We have

$$
v=\sum_{j \geq 1} v_{j}(t) w_{j}(x)
$$


and

$$
y=\sum_{j \geq 1} \int_{0}^{t} e^{-\left(\lambda_{j}-k \lambda_{j}^{\theta}\right)(t-s)} v_{j}(s) d s w_{j}(x) .
$$

Therefore equation (4.11) becomes

$$
\int_{0}^{T} e^{-\left(\lambda_{j}-k \lambda_{j}^{\theta}\right)(T-s)} v_{j}(s) d s=\int_{0}^{T} e^{-\lambda_{j}(T-s)} p_{j}(s) d s
$$

The simplest solution of (4.13) is given by

$$
v_{j}(s)=e^{-k \lambda_{j}^{\theta}(T-s)} p_{j}(s) .
$$

Thus, the control

$$
v=\sum_{j \geq 1} e^{-k \lambda_{j}^{\theta}(T-s)} p_{j}(s) w_{j}(x)
$$

is such that (4.2) holds and consequently

$$
\mathcal{M}\left(k ; 0, y^{1}+\beta B\right) \leq \frac{1}{2} \int_{0}^{T} \int_{\Omega} v^{2}=\frac{1}{2} \sum_{j \geq 1} \int_{0}^{T} e^{-2 k \lambda_{j}^{\theta}(T-s)} p_{j}^{2}(s) d s
$$

Thus, it is sufficient to see that

$$
\sum_{j \geq 1} \int_{0}^{T} e^{-2 k \lambda_{j}^{\theta}(T-s)} p_{j}^{2}(s) d s \rightarrow 0, \text { as } k \rightarrow \infty .
$$

This can be done easily. First of all we observe that $\int_{0}^{T} e^{-2 k \lambda_{j}^{\theta}(T-s)} p_{j}^{2}(s) \rightarrow 0$ as $k \rightarrow \infty$ for each $j \in N$ by Lebesgue's dominated convergence Theorem. Indeed, we have:

(a) $e^{--2 k \lambda_{j}^{\theta}(T-s)} p_{j}^{2}(s) \rightarrow 0$ a.e. $s \in(0, T)$ as $k \rightarrow \infty$;

(b) $e^{-2 k \lambda_{j}^{\theta}(T-s)} p_{j}^{2}(s) \leq p_{j}^{2}(s)$ a.e. $s \in(0, T), \forall k>0$.

The fact that (4.16) holds follows also by Lebesgue's Theorem since

$$
\int_{0}^{T} e^{-2 k \lambda_{j}^{\theta}(T-s)} p_{j}^{2}(s) d s \leq \int_{0}^{T} p_{j}^{2}(s) d s, \forall k>0, \forall j \in \mathbb{N}
$$


and

$$
\sum_{j \geq 1} \int_{0}^{T} p_{j}^{2}(s) d s=\int_{0}^{T} \int_{\Omega} p^{2} d x d t<\infty .
$$

We have proved the following:

Proposition 4.1. When $\omega=\Omega$ for every $y^{1} \in L^{2}(\Omega)$ and $\beta>0$,

$$
\mathcal{M}\left(k ; 0, y^{1}+\beta B\right) \rightarrow 0 \text { as } k \rightarrow \infty .
$$

Remark 4.1. The method of proof of Proposition 4.1 does not apply when the support of the control is restricted to some strict open subset $\omega$ of $\Omega$. Indeed, the method fails since even if $\operatorname{supp}(p) \subset \omega \times(0, T)$, we can not guarantee that the control $v$ given by (4.14) satisfies $\operatorname{supp}(v) \subset$ $\omega \times(0, T)$ except when $\theta=0$.

The case $\theta=0$ is the object of the following example.

Example 2: $\theta=0$.

Let us consider the particular case $\theta=0$ but not for any open subset $\omega$ of $\Omega$.

The system under consideration is:

$$
\left\{\begin{array}{lll}
y^{\prime}-\Delta y-k y=v 1_{\omega} & \text { in } & Q \\
y=0 & \text { on } & \Sigma \\
y(0)=0 & \text { in } & \Omega .
\end{array}\right.
$$

As in example 1 above we consider first

$$
\left\{\begin{array}{lll}
z^{\prime}-\Delta z=p 1_{\omega} & \text { in } & Q \\
z=0 & \text { on } & \Sigma \\
z(0)=0 & \text { in } & \Omega
\end{array}\right.
$$

Given $y^{1} \in L^{2}(\Omega)$ and $\beta>0$ we choose $p$ as being the optimal control for (4.18) such that $z(T) \in y^{1}+\beta B$.

We then look for $v$ such that the solution of (4.17) satisfies (4.11). Proceeding as in Example 1 above we obtain

$$
v^{k}=\sum_{j \geq 1} e^{-k(T-s)} p_{j}(s) w_{j}(x)=e^{-k(T-s)} p(x, s)
$$


We see that, $\operatorname{since} \operatorname{supp}(p) \subset \omega \times(0, T)$ we also have $\operatorname{supp}(v) \subset \omega \times(0, T)$.

By Lebesgue's Theorem it is easy to see that $v^{k} \rightarrow 0$ in $L^{2}(\omega \times(0, T))$ and therefore (4.17) holds too.

We have proved the following result:

Proposition 4.2. Let $\omega$ be any open non-empty subset of $\Omega$. Suppose that $\theta=0$. Then, for any $y^{1} \in L^{2}(\Omega)$ and $\beta>0$, (4.17) holds.

\section{Controlling finite-dimensional projections from $y^{0} \equiv 0$}

\subsection{The case where the number of frequencies is fixed}

We introduce the following family of finite-dimensional subspaces of $L^{2}(\Omega)$ :

$$
E_{\mu}=\operatorname{span}\left\{w_{j}: \lambda_{j} \leq \mu\right\} .
$$

By $\pi_{\mu}$ we denote the orthogonal projection from $L^{2}(\Omega)$ on $E_{\mu}$.

We consider the system:

$$
\left\{\begin{array}{lll}
y^{\prime}-\Delta y-k(-\Delta)^{\theta} y=v 1_{\omega} & \text { in } & Q \\
y=0 & \text { on } & \Sigma \\
y(0)=0 & \text { in } & \Omega
\end{array}\right.
$$

and given $y^{1} \in L^{2}(\Omega)$ and $\mu>0$ we analyze the controls $v$ such that

$$
\pi_{\mu}\left(y(T)-y^{1}\right)=0 .
$$

We introduce the set of admissible controls

$$
\mathcal{U}_{a d}^{k}\left(0, \pi_{\mu}\left(y^{1}\right)\right)=\left\{v \in L^{2}(Q): y \text { solution of (5.2) satisfies (5.3) }\right\} \text {. }
$$

The admissible set $\mathcal{U}_{a d}^{k}$ is non-empty. Indeed, as we have seen in section 4 above, the reachable set $\left\{y(T): v \in L^{2}(Q)\right\}$ is dense in $L^{2}(\Omega)$. Consequently, for any $y^{1} \in L^{2}(\Omega)$ and $\varepsilon>0$ there exists $v \in L^{2}(Q)$ such that

$$
\left\{\begin{array}{l}
\left\|y(T)-y^{1}\right\|_{L^{2}(\Omega)} \leq \varepsilon \\
\pi_{\mu}\left(y(T)-y^{1}\right)=0
\end{array}\right.
$$

(see Appendix B for the details of the proof and [Z] for related questions). 
Observe that we do not address here the control problem with the final conditions (5.5) but rather relax those final requirements to (5.3) in which we only take into account the finite-dimensional projection of $y(T)$ over $E_{\mu}$.

However the existence of controls such that (5.5) holds guarantees that the admissible set of controls $\mathcal{U}_{a d}^{k}\left(0, \pi_{\mu}\left(y^{1}\right)\right)$ is non-empty.

Let us analyze now the cost of controlling this finite-dimensional projection in terms of the parameter $k$, i.e. the quantity

$$
\mathcal{M}\left(k ; 0, \pi_{\mu}\left(y^{1}\right)\right)=\inf _{v \in \mathcal{U}_{a d^{l}}^{k}\left(0, \pi_{\mu}\left(y^{1}\right)\right)} \frac{1}{2} \int_{0}^{T} \int_{\omega} v^{2} d x d t .
$$

We have the following result:

Theorem 5.1. For any open subset $\omega$ of $\Omega, \mu>0, \theta>0$ and $y^{1} \in L^{2}(\Omega)$ it follows that

$$
\lim _{k \rightarrow \infty} \mathcal{M}\left(k ; 0, \pi_{\mu}\left(y^{1}\right)\right)=0 .
$$

As an immediate corollary of Theorem 5.1 the following can be said for the case in which $y^{0} \not \equiv 0$, i.e. for system

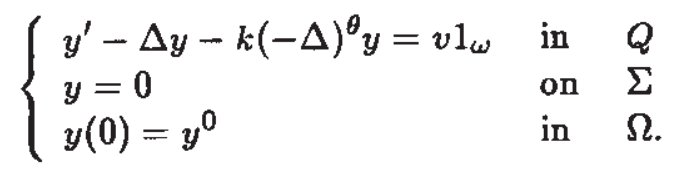

Given $\mu>0$ and $y^{1} \in L^{2}(\Omega)$ we introduce the set of admissible controls

$$
\begin{aligned}
\mathcal{U}_{a d}^{k}\left(y^{0}, \pi_{\mu}\left(y^{1}\right)\right)= & \left\{v \in L^{2}(Q):\right. \\
& y \text { solution of (5.8) satisfies (5.3) }\}
\end{aligned}
$$

and the cost of achieving the control property (5.3), $\mathcal{M}\left(k ; y^{0}, \pi_{\mu}\left(y^{1}\right)\right)$, as

$$
\mathcal{M}\left(k ; y^{0}, \pi_{\mu}\left(y^{1}\right)\right)=\inf _{v \in \mathcal{U}_{a d}^{k}\left(y^{0}, \pi_{\mu}\left(y^{1}\right)\right)} \frac{1}{2} \int_{0}^{T} \int_{\omega} v^{2} d x d t .
$$

As a consequence of Theorem 5.1 the following holds: 
Corollary 5.1. Under the assumptions of Theorem 5.1, if $y^{0} \in L^{2}(\Omega)$ is such that $\pi_{\mu}\left(y^{0}\right)=0$ then

$$
\mathcal{M}\left(k ; y^{0}, \pi_{\mu}\left(y^{1}\right)\right) \rightarrow 0 \text { as } k \rightarrow \infty .
$$

Remark 5.1. Theorem 5.1 guarantees that the cost of controlling exactly a finite number of frequencies converges to zero as the parameter $k$ measuring the degree of unstability of the system diverges. However, this holds when the initial data vanishes, or, as in Corollary 5.1, when the projection of the initial data over the frequencies to be controlled vanishes.

Remark 5.2. The same control problem for system (5.8) can also be addressed when $\pi_{\mu}\left(y^{0}\right) \neq 0$. In this case the same proof of Theorem 3.1 applies and we obtain that $\mathcal{M}\left(k ; y^{0}, \pi_{\mu}\left(y^{1}\right)\right) \rightarrow \infty$ as $k \rightarrow \infty$.

Therefore we see that the unstability may make more expensive controlling a finite-dimensional projection. This depends on whether our control problem is compatible with the dynamics of the system in which the energy of every Fourier component of the system, in the absence of control, increases more and more rapidly as $k \rightarrow \infty$.

Remark 5.3. The proof of Theorem 5.1 provides an estimate of how rapidly $\mathcal{M}\left(k ; 0, \pi_{\mu}\left(y^{1}\right)\right) \rightarrow 0$. Indeed from (5.11) below it is easy to see that for every $y^{1} \in L^{2}(\Omega)$ there exists a constant $C>0$ such that

$$
\mathcal{M}\left(k ; 0, \pi_{\mu}\left(y^{1}\right)\right) \leq C \frac{k}{e^{2 \lambda_{1}^{\theta} T k}}, \text { as } k \rightarrow \infty .
$$

In fact, if $\pi_{\mu^{\prime}}\left(y^{1}\right)=0$ for some $\mu^{\prime}=\lambda_{j_{0}}<\mu$ this estimate can be improved and

$$
\mathcal{M}\left(k ; 0, \pi_{\mu}\left(y^{1}\right)\right) \leq C \frac{k}{e^{2 \lambda_{j 0+1}^{\theta}} \overline{T k}} \text { as } k \rightarrow \infty
$$

holds for a suitable $C>0$. 
Remark 5.4. The understanding of Theorem 5.1 and of the estimate of Remark 5.3 can be improved by looking at the case $k \rightarrow-\infty$, i.e. when we want to control finite dimensional projections from $y^{0}=0$ of operators which are more and more stable.

We shall prove in section 5.3 that

$$
\mathcal{M}\left(k ; 0, \pi_{\mu}\left(y^{1}\right)\right) \rightarrow+\infty \text { as fast as }|k| \text { when } k \rightarrow-\infty \text {. }
$$

Assuming that Theorem 5.1 holds let us prove Corollary 5.1:

\section{Proof of Corollary 5.1.}

We decompose the solution $y$ of (5.8) as $y=y_{0}+z$ where $y_{0}$ solves

$$
\left\{\begin{array}{lll}
y_{0}^{\prime}-\Delta y_{0}-k(-\Delta)^{\theta} y_{0}=0 & \text { in } & Q \\
y_{0}=0 & \text { on } & \Sigma \\
y_{0}(0)=y^{0} & \text { in } & \Omega
\end{array}\right.
$$

and

$$
\left\{\begin{array}{lll}
z^{\prime}-\Delta z-k(-\Delta)^{\theta} z=v 1_{\omega} & \text { in } & Q \\
z=0 & \text { on } & \Sigma \\
z(0)=0 & \text { in } & \Omega
\end{array}\right.
$$

Then $\pi_{\mu}\left(y(T)-y^{1}\right)=0$ if and only if $\pi_{\mu}\left(z(T)-\left(y^{1}-y_{0}(T)\right)\right)=$ 0 . However, since $\pi_{\mu}\left(y^{0}\right)=0, \pi_{\mu}\left(y_{0}(T)\right)=0$ too and therefore this condition is equivalent to $\pi_{\mu}\left(z(T)-y^{1}\right)=0, \mathcal{M}\left(k ; 0, \pi_{\mu}\left(y^{1}\right)\right)=$ $\mathcal{M}\left(k ; y^{0}, \pi_{\mu}\left(y^{1}\right)\right)$ and consequently (5.11) holds.

Proof of Theorem 5.1. First of all we observe that the optimal control realizing the minimum (5.6) among the admissible controls can be characterized by means of the adjoint system

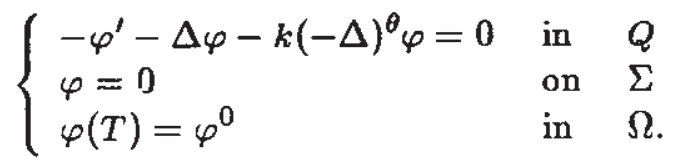


More precisely

$\left\{\begin{array}{l}v=\varphi \text { where } \varphi \text { solves }(5.8) \text { with } \varphi^{0} \in E_{\mu} \text { such that } \\ \text { the functional } J_{\mu}^{k} \text { below achieves its minimum in } E_{\mu}\end{array}\right.$

$$
J_{\mu}^{k}\left(\varphi^{0}\right)=\frac{1}{2} \int_{0}^{T} \int_{\omega} \varphi^{2} d x d t-\int_{\Omega} y^{1} \varphi^{0} d x .
$$

On the other hand

$$
\mathcal{M}\left(k ; 0, \pi_{\mu}\left(y^{1}\right)\right)=-\min _{\varphi^{0} \in E_{\mu}} J_{\mu}^{k}\left(\varphi^{0}\right) .
$$

We refer to [Lil] and [Z] for the details in the case of the classical heat equation.

We recall that according to Lemma 3.1:

$$
\int_{\omega}\left|\sum_{\lambda_{j} \leq \mu} a_{j} w_{j}(x)\right|^{2} d x \geq C_{1} e^{-C_{2} \sqrt{\mu}} \sum_{\lambda_{j} \leq \mu}\left|a_{j}\right|^{2}, \forall\left\{a_{j}\right\} \in \ell^{2} .
$$

Actually since the frequency $\mu$ is fuxed in Theorem 5.1 , it suffices to observe that there exists $C>0$ such that

$$
\int_{\omega}\left|\sum_{\lambda_{j} \leq \mu} a_{j} w_{j}(x)\right|^{2} d x \geq C \sum_{\lambda_{j} \leq \mu}\left|a_{j}\right|^{2}, \forall\left\{a_{j}\right\} \in \ell^{2}
$$

which follows from the fact that the $w_{j}$ 's restricted to $\omega$ are linearly independent in $L^{2}(\omega)$ when $\lambda_{j} \leq \mu$.

On the other hand. any solution $\varphi$ of (5.15) with data $\varphi^{0} \in E_{\mu}$ can be written as

$$
\varphi(x, t)=\sum_{\lambda_{j} \leq \mu} a_{j} e^{\left(k \lambda_{j}^{\theta}-\lambda_{j}\right)(T-t)} w_{j}(x)
$$

provided

$$
\varphi^{0}=\sum_{\lambda_{j} \leq \mu} a_{j} w_{j}(x)
$$

Therefore

$$
J_{\mu}^{k}\left(\varphi^{0}\right)=\frac{1}{2} \int_{0}^{T} \int_{\omega}\left|\sum_{\lambda_{j} \leq \mu} a_{j} e^{\left(k \lambda_{j}^{\theta}-\lambda_{j}\right)(T-t)} w_{j}\right|^{2} d x d t
$$




$$
\begin{aligned}
& -\sum_{\lambda_{j} \leq \mu} a_{j} \int_{\Omega} y^{1} w_{j} d x \\
& \geq \frac{c}{2} \int_{0}^{T} \sum_{\lambda_{j} \leq \mu}\left|a_{j}\right|^{2} e^{2\left(k \lambda_{j}^{\theta}-\lambda_{j}\right)(T-t)} d t-\sum_{\lambda_{j} \leq \mu} a_{j} \int_{\Omega} y^{1} w_{j} d x \\
& =\frac{c}{2} \sum_{\lambda_{j} \leq \mu}\left|a_{j}\right|^{2}\left(\frac{e^{2\left(k \lambda_{j}^{\theta}-\lambda_{j}\right) T}-1}{2\left(k \lambda_{j}^{\theta}-\lambda_{j}\right)}\right)-\sum_{\lambda_{j} \leq \mu} a_{j} \int_{\Omega} y^{1} w_{j} d x .
\end{aligned}
$$

We set.

$$
\alpha_{j}=C\left(\frac{e^{2\left(k \lambda_{j}^{\theta}-\lambda_{j}\right) T}-1}{2\left(k \lambda_{j}^{\theta}-\lambda_{j}\right)}\right) .
$$

Then

$$
\begin{aligned}
-\mathcal{M}\left(k ; 0, \pi_{\mu}\left(y^{1}\right)\right) & \geq \inf _{\left\{a_{j}\right\}_{\lambda_{j} \leq \mu}}\left[\frac{1}{2} \sum_{\lambda_{j} \leq \mu} \alpha_{j} a_{j}^{2}-\sum_{\lambda_{j} \leq \mu} a_{j} \int_{\Omega} y^{1} w_{j} d x\right] \\
& =-\frac{1}{2} \sum_{\lambda_{j} \leq \mu} \frac{\left(\int_{\Omega}\right)^{2}}{\alpha_{j}} .
\end{aligned}
$$

Thus

$$
\mathcal{M}\left(k ; 0, \pi_{\mu}\left(y^{1}\right)\right) \leqq \frac{1}{2} \sum_{\lambda_{j} \leq \mu} \frac{\left(\int_{\Omega} y^{1} w_{j} d x\right)^{2}}{\alpha_{j}} .
$$

On the other hand, for every $j$ fixed, $\alpha_{j} \rightarrow \infty$ as $k \rightarrow \infty$. Therefore the upper bound on (5.20) converges to zero as $k \rightarrow \infty$ and (5.7) holds.

\subsection{The case where the number of frequencies increases.}

In section 5.1 we have adressed the problem of controlling a finite number of frequencies that is independent of $k$. However the same problem can be considered when $\mu=\mu(k) \rightarrow \infty$ as $k \rightarrow \infty$. In fact one would like to know how rapidly may $\mu(k)$ grow and still keep the property that the cost of controlling converges to zero as $k \rightarrow \infty$. 
The following result provides an answer to this problem:

Theorem 5.2. Under the assumptions of Theorem 5.1, if

$$
\mu(k)=\delta k^{\sigma}
$$

for some $\delta>0$ small enough and $\sigma=\min (2,1 /(1-\theta))$, it follows that

$$
\mathcal{M}\left(k ; 0, \pi_{\mu(k)}\left(y^{1}\right)\right) \rightarrow 0 \text { as } k \rightarrow \infty
$$

Remark 5.5. Observe that the frequencies that are unstabilized by $k$ are those for which

$$
-\lambda_{j}+k \lambda_{j}^{\theta}>0
$$

i.e. $\lambda_{j}^{1-\theta}<k$ or $\lambda_{j}<k^{1 /(1-\theta)}$. In view of $(5.20)$ we have $\mu(k)=\delta k^{\sigma}<<$ $k^{1 /(1-\theta)}$ as $k \rightarrow \infty$ when $\theta>1 / 2$. Therefore the range of frequencies in which Theorem 5.2 applies does not cover the whole range of unstable frequencies for $\theta>1 / 2$.

Whether (5.22) holds with $\mu(k)=\delta k^{\sigma}$ for any $\sigma<1 /(1-\theta)$ or not when $\theta>1 / 2$ is an open problem.

The iterative arguments developed in $[\mathrm{LR}]$ and $[\mathrm{LZ}]$ for the nullcontrollability of the heat equation that are based on Lemma 3.1 may be helpful to address this problem.

Proof of Theorem 5.2. As in the proof of Theorem 5.1 (see (5.19)) we see that

$$
\mathcal{M}\left(k ; 0, \pi_{\mu(k)}\left(y^{1}\right)\right) \leq \frac{1}{2} \sum_{\lambda_{j} \leq \mu(k)} \frac{\left(\int_{\Omega} y^{1} w_{j} d x\right)^{2}}{\alpha_{j}}
$$

with

$$
\alpha_{j}=C_{1} e^{-C_{2} \sqrt{\mu(k)}}\left(\frac{e^{\left(k \lambda_{j}^{\theta}-\lambda_{j}\right)^{T}}-1}{2\left(k \lambda_{j}^{\theta}-\lambda_{j}\right)}\right) .
$$

In view of $(5.21)$ we see that

$$
2 T\left(k \lambda_{j}^{\theta}-\lambda_{j}\right)>C_{3} \sqrt{\mu(k)}
$$


with $C_{3}>C_{2}$ provided

$$
C_{3} \sqrt{\delta} k^{\sigma / 2}+2 T \lambda_{j} \leq 2 T k \lambda_{j}^{\theta}
$$

which holds if

$$
\begin{cases}C_{3} \sqrt{\delta} k^{\sigma / 2} & \leq T k \lambda_{j}^{\theta} \\ 2 \lambda_{j} & \leq k \lambda_{j}^{\theta} .\end{cases}
$$

The second inequality of (5.27) holds if $\lambda_{j} \leq\left(\frac{k}{2}\right)^{1 /(1-\theta)}$. The first one holds provided $\sigma=2$ and $\delta \leq\left(T \lambda_{1}^{\theta} / C_{3}\right)^{2}$. Therefore if $\mu(k)$ is as in (5.21) with $\sigma=\min (2,1 /(1-\theta))$ and $\delta$ small enough we deduce that (5.25) holds.

Going back to (5.24) and taking into account that the function $x \mapsto$ $\left(e^{x}-1\right) / x$ is increasing for $x \geq 1$ we deduce that

$$
\alpha_{j} \geq C e^{-C_{2} \sqrt{\mu(k)}} \frac{e^{C_{3} \sqrt{\mu(k)}}-1}{\sqrt{\mu(k)}}=C \frac{e^{\left(C_{3}-C_{2}\right) \sqrt{\mu(k)}}-e^{-C_{2} \sqrt{\mu(k)}}}{\sqrt{\mu(k)}}
$$

Combining (5.23) and (5.28) we deduce that

$$
\begin{aligned}
\mathcal{M}\left(k ; \mathbf{0}, \pi_{\mu(k)}\left(y^{1}\right)\right) & \leq C \frac{\sqrt{\mu(k)}}{\left(e^{\left(C_{3}-C_{2}\right) \sqrt{\mu(k)}}-e^{-C_{2} \sqrt{\mu(k)}}\right)} \sum_{\alpha_{j} \leq \mu(k)}\left(\int_{\Omega} y^{1} w_{j} d x\right)^{2} \\
& \leq C \frac{\sqrt{\mu(k)}}{\left(e^{\left(C_{3}-C_{2}\right) \sqrt{\mu(k)}}-e^{-C_{2} \sqrt{\mu(k)}}\right)}\left\|y^{1}\right\|_{L^{2}(\Omega)}^{2} \rightarrow 0
\end{aligned}
$$

as $k \rightarrow \infty$ since $C_{3}>C_{2}$.

Remark 5.6. Observe that the proof of Theorem 4.2 shows that $\mathcal{M}\left(k ; 0, \pi_{\mu(k)}\left(y^{1}\right)\right)$ converges exponentially to zero.

\subsection{The case where the operator becomes more and more} stable.

Following Remark 5.4, we consider the case where $k \rightarrow-\infty$ in (5.2). Of course (5.17) is still valid. We choose a particular set of $\varphi^{0}$, namely

$$
\left\{\begin{array}{l}
\rho^{0}=\rho w_{j}, \rho \in \mathbb{R} \text { to be chosen, } \\
\text { where } j \text { is such that } \int_{\Omega} y^{1} w_{j}=a_{j} \neq 0 .
\end{array}\right.
$$


Then

$$
\inf _{\varphi^{0}} J_{\mu}^{k}\left(\varphi^{0}\right) \leq \inf _{\rho \in \mathbb{R}} J_{\mu}^{k}\left(\rho w_{j}\right)
$$

But for the choice (5.30) one has

$$
\left\{\begin{array}{l}
\varphi=\rho w_{j} \varphi_{j} \\
-\varphi_{j}^{\prime}+\left(\lambda_{j}-k \lambda_{j}^{\theta}\right) \varphi_{j}=0, \varphi_{j}(T)=1,
\end{array}\right.
$$

i.e. $\varphi_{j}=e^{-\left(\lambda_{j}-k \lambda_{j}^{\theta}\right)(T-t)}$.

If we set

$$
\alpha_{j}=\left(\int_{\omega} w_{j}^{2} d x\right) \int_{0}^{T} \varphi_{j}^{2} d t
$$

then

$$
J_{\mu}^{k}\left(\rho w_{j}\right)=\frac{1}{2} \alpha_{j} \rho^{2}-a_{j} \rho
$$

so that

$$
\inf J_{\mu}^{k}\left(\rho w_{j}\right)=-\frac{a_{j}^{2}}{2 \alpha}
$$

Using (5.17), (5.31), (5.34) we obtain

$$
\mathcal{M}\left(k ; 0, \pi_{\mu}\left(\dot{y}^{1}\right)\right) \geq \frac{a_{j}^{2}}{2 \alpha_{j}} .
$$

When $k \rightarrow-\infty, 1 / \alpha_{j}$ increases like $|k|$, hence the result of Remark 5.4 follows.

We have obtained, in conclugion, that $\mathcal{M}\left(k ; 0, \pi_{\mu}\left(y^{1}\right)\right)$ goes to zero when the system gets more and more unstable $(k \rightarrow+\infty)$ and goes to $+\infty$ when the system gets more and more stable $(k \rightarrow-\infty)$.

\section{Further comments}

The results of section 3 and 4 apply in the more general setting of abstract parabolic equation of the form

$$
y^{\prime}+A y-k A^{\theta} y=v 1_{\omega}
$$

where $A$ is an unbounded, self-adjoint, coercive operator in the Hilbert space $H\left(=L^{2}(\Omega)\right.$ or $\left(L^{2}(\Omega)\right)^{n}$ in most examples $), A^{-1}$ being compact. 
The results of section 5 do not apply in this general context. Indeed, as far as we know, the most general result analogous to Lemma 4.1 is due to L. Escauriaza $[E]$ and it applies to second order elliptic symmetric operators in divergence form with Lipschitz coefficients in Hölder domains. Therefore, in principle, the results of section 5 are valid for this case.

However, the proof of Theorem 5.1 requires a result much weaker than Lemma 5.1. In fact, the proof of Theorem 4.1 only requires the following uniqueness result:

$$
\left\{\begin{array}{l}
\text { If }\left\{a_{j}\right\}_{\lambda_{j} \leq \mu} \text { are such that } \sum_{\lambda_{j} \leq \mu} a_{j} w_{j}=0 \text { in } \omega, \\
\text { then } a_{j}=0 \text { for all } \lambda_{j} \leq \mu .
\end{array}\right.
$$

Indeed, if (6.2) holds, taking into account that the space of elements of the form $\sum_{\lambda_{j} \leq \mu} a_{j} w_{j}$ is finite-dimensional, for any $0<\mu<\infty$ we deduce the existence of a positive constant $C(\mu)>0$ such that

$$
\int_{\omega}\left|\sum_{\lambda_{j} \leq \mu} a_{j} w_{j}\right|^{2} d x \geq C(\mu) \sum_{\lambda_{j} \leq \mu}\left|a_{j}\right|^{2}, \forall\left\{a_{j}\right\} .
$$

However, obviously, this uniqueness argument does not provide any estimate on the growth of $C(\mu)$ with respect to $\mu$.

Therefore, we see that the analogous of Theorem 5.1, i.e. controlling a fixed finite number of frequencies, only needs the uniqueness result of (6.2)

The uniqueness result of (6.2) holds for a large class of elliptic operators $A$. Indeed, proceeding as in [LR] and [LZ] we set

$$
p(t)=\sum_{\lambda_{j} \leq \mu} a_{j} \frac{\sinh \left(\sqrt{\lambda_{j}} t\right)}{\sqrt{\lambda_{j}}} w_{j} .
$$

Then $p$ solves the elliptic system

$$
\left\{\begin{array}{l}
p^{\prime \prime}-A p=0 \\
p(t) \in D(A), 0<t<T \\
p(0)=0
\end{array}\right.
$$

On the other hand $p^{\prime}(0)=\sum_{\lambda_{j} \leq \mu} a_{j} w_{j}$. Therefore (6.2) holds whenever the elliptic unique continuation result applies to the operator $\partial_{t}^{2}-A$ 
in $\Omega \times(0, T)$. In particular, it holds for elliptic operators $A$ of order $2 m, m \geq 1$ and also for Stokes operator.

More precisely, we can consider, for instance, the system

$$
\left\{\begin{array}{lll}
y^{\prime}+\Delta^{2} y+k\left(\Delta^{2}\right)^{\theta} y=v 1_{\omega} & \text { in } & Q \\
y=\Delta y=0 & \text { on } & \Sigma \\
y(0)=y^{0} & \text { in } & \Omega .
\end{array}\right.
$$

In this case, all the results of this paper apply, since the eigenfunctions of the underlying elliptic operator are precisely those of the Dirichlet Laplacian and therefore Lemma 3.1 applies.

The situation is different when the boundary conditions of $(6.5)$ are replaced by

$$
y=\partial y / \partial \nu=0 \text { on } \Sigma \text {. }
$$

To our knowledge it is unknown whether the analogous of Lemma 3.1 holds for the bilaplacian with these boundary conditions. Therefore we do not know if Theorem 5.2 applies in this case. The rest of the results of this paper, and in particular, Theorem 5.1 apply in this case since the uniqueness property (6.2) holds. Indeed, proceeding as above the problem is reduced to the unique continuation in (6.5). Iu this case, since the coefficients of the operaror $A$ are constant, Holmgren's uniqueness Theorem can be applied.

The situation is the same for the Stokes system. Indeed, all but Theorem 5.2 of the results of this paper apply. It is unknown whether the analogous of Lemma 3.1 holds for the eigefunctions of the Stokes system too.

\section{References}

[BLR] C. Bardos, G. Lebeau and J. Rauch, Sharp sufficient conditions for the observation, control and stabilization of waves from the boundary, SIAM J. Cont. Optim., 30 (1992), 1024-1065.

[CMZ] G. Chen, Ph. J. Morris and J. Zhow, Visualization of Special Eigenmode Shapes of a Vibrating Elliptical Membrane, SIAM Rev., 36 (3) (1994), 453-469.

[E] L. Escauriaza, private communication. 
[FPZ] C. Fabre, J. P. Puel and E. Zuazua, Approximate controllability of the semilinear heat equation, Proc. Roy. Soc. Edinburgh, 125 A (1995), 31-61.

[GLi] R. Glowinski and J.-L. Lions, to appear.

[LR] G. Lebeau and L. Robbiano, Contrôle exact de l'équation de la chaleur, Comm. PDE, 20 (1995), 335-356.

[LZ] G. Lebeau and E. Zuazua, Null controllability of a system of thermoelasticity, Archives for Rational Mechanics and Analysis, to appear.

[Li1] J.-L. Lions, Contrôlabilité exacte, stabilisation et perturbations de Systèmes Distribués. Tome 1. Contrôlabilité exacte \& Tome 2. Perturbations, RMA 8 \& 9, Masson, Paris, 1988.

[Li2] J.-L. Lions, Remarks on approximate controllability, J. Analyse Math., 59 (1992), 103-116.

[Li3] J.-L. Lions, Measures of controllability, Proc. of the Georgian Academy of Sciences Math., 1 (1) (1993), 53-59.

[LiM] J.-L. Lions and E. Magenes, Problèmes aux limites non homogènes et applications. Vol, 3, Dunod, Paris, 1970.

[LiZ1] J.-L. Lions and E. Zuazua, Approximate controllability for a coupled hydro-elastic system, ESAIM: COCV, 1(1) (1995), 1-15 (http://www.emath.fr/cocv/).

[LiZ2] J.-L. Lions and E. Zuazua, A generic uniqueness result for the Stokes system and its control theoretical consequences, in Partial Differential Equations and Applications, P. Marcellini, G. Talenti and E. Visentini eds., Marcel-Dekker Inc., LNPAS \# 177, 1996, 221-235.

[LiZ3] J.-L. Lions and E. Zuazua, The cost of controlling unstable systems: The case of time reversible systems, to appear.

[LiZ4] J.-L. Lions and E. Zuazua, Contrôlabilité exacte des approximations de Galerkin des équations de Navier-Stokes, C. R. Acad. Sci. Paris, 234 (1997), 1015-1021. 
[LiZ5] J.-L. Lions and E. Zuazua, The cost of controlling unstable systems: the case of boundary controls, J. Anal Math., to appear.

[P] A. Pazy, Semigroups of linear operators and applications to partial differential equations, Applied Mathematical Sciences 34, Springer, 1983.

[Z] E. Zuazua, Finite dimensional null controllability for the semilinear heat equation, J. Math. pures et appl., 76 (1997), 237-264.

\section{Appendix A:}

The object of this appendix is to give the details of the proof the uniqueness result for system (3.3) stated in the beginning of section 4 .

More precisely, consider the system

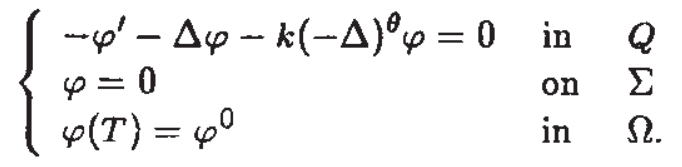

Then, the following holds:

Proposition A. Let $\omega$ be an open and non-empty subset of $\Omega$ and $T>0$. Assume that $k>0$ and $0 \leq \theta<1$. Let $\varphi$ be a solution of (A.1) with $\varphi^{0} \in L^{2}(\Omega)$ and such that

$$
\varphi \equiv 0 \text { in } \omega \times(0, T) .
$$

Then necessarily $\varphi \equiv 0$ everywhere, i.e. $\varphi^{0} \equiv 0$.

\section{Proof of Proposition A.}

By the change of variables $t \rightarrow T-t$, system (A.1) is reduced to

$$
\left\{\begin{array}{lll}
\psi^{\prime}-\Delta \psi-k(-\Delta)^{\theta} \psi=0 & \text { in } & Q \\
\psi=0 & \text { on } & \Sigma \\
\psi(0)=\varphi^{0} & \text { in } & \Omega .
\end{array}\right.
$$

The semigroup generated by the operator $-\Delta-k(-\Delta)^{\theta}$ is analytic. On the other hand, due to the regularizing effect of system (A.3), for any $\tau>0$ and $s>0$, the solution $\psi$ of (A.3) is such that $\psi(s) \in$ $\mathcal{D}\left(\left(-\Delta-k(-\Delta)^{\theta}\right)^{s}\right)$. 
By elliptic regularity we deduce that, for any $x_{0} \in \Omega, \psi\left(x_{0}, t\right)$ is analytic for $t>0$.

In view of (A.2) and this analyticity property we deduce that

$$
\psi \equiv 0 \text { in } \omega \times(0, \infty) .
$$

On the other hand, $\psi$ can be developed in Fourier series

$$
\psi=\sum_{j \in \mathbb{N}} a_{j} e^{-\mu_{j} t} w_{j}(x)
$$

where $\left\{w_{j}\right\}$ is an orthonormal basis of $L^{2}(\Omega)$ constituted by the eigenfunctions of the Laplacian and $\mu_{j}=\lambda_{j}-k \lambda_{j}^{\theta}$ where $\lambda_{j}$ are the eigenvalues of $-\Delta$.

Let $j_{0}$ be such that

$$
\mu_{j_{0}}=\min _{j \in \mathbb{N}}\left(\mu_{j}\right) .
$$

Then, multiplying in (A.5) by $e^{\mu_{j_{0}} t}$ we observe that

$$
e^{\mu_{0} t} \psi=a_{j_{0}} w_{j_{0}}(x)+\sum_{\substack{j \in \mathbb{N} \\ j \neq j_{0}}} a_{j} e^{-\left(\mu_{j}-\mu_{j_{0}}\right) t} w_{j}
$$

Combining (A.6) with (A.4) and the fact that the last term of (A.6) converges to zero in $L^{2}(\Omega)$ as $t \rightarrow \infty$, we deduce that

$$
a_{j_{0}} w_{j_{0}} \equiv 0 \text { in } \omega
$$

Then, either $a_{j 0}=0$ or

$$
w_{j_{0}} \equiv 0 \text { in } \omega .
$$

However, (A.8) may not hold by elliptic unique-continuation, since $w_{j_{0}}$ is an eigenfunction of $-\Delta$. Thus $a_{j_{0}}=0$.

Therefore

$$
\psi=\sum_{\substack{j \in \mathbb{N} \\ j \neq j_{0}}} a_{j} e^{-\mu_{j} t} w_{j}
$$

In a second step we consider $j_{1} \in \mathbb{N}$ such that

$$
\mu_{j_{1}}=\min _{\substack{j \in \mathbb{N} \\ j \neq j_{0}}}\left(\mu_{j}\right)
$$


Proceeding as above we see that $a_{j_{1}}=0$ too.

By inducting we prove that $a_{j}=0$ for all $j \in \mathbb{N}$.

This concludes the proof of the Proposition.

\section{Appendix B:}

This section is devoted to prove, as indicated in section 5.1 , that approximate controllability implies the simultaneous finite-approximate controllability property (5.5). This is in fact a consequence of the following abstract result:

Theorem. Let $V$ and $H$ be two Hilbert spaces and $L$ a bounded linear operator from $V$ to $H$ with dense range. Let $E$ be a finite-dimensional subspace of $H$ and $\Pi_{E}$ the corresponding orthogonal projection.

Then, given any $e_{0} \in E$, when $v$ runs over the set of elements of $V$ such that

$$
\Pi_{E} L v=e_{0},
$$

$L v$ describes a dense set in $e_{0}+E^{\perp}$.

Proof. We identify $H$ with its dual and denote by $(\cdot, \cdot)$ both the scalar product in $H$ and the duality pairing between $V$ and $V^{\prime}$.

We observe that, since $L$ is of dense range, its adjoint $L^{*} \in \mathcal{L}\left(H ; V^{\prime}\right)$, $V^{\prime}$ being the dual of $V$, is injective, i. e. $L^{*} h=0$ implies that $h=0$.

We note that it is sufficient to prove the lemma when $e_{0}=0$. Indeed, given $e_{0}$ in $E$ it is easy to find an element $v_{0}$ in $V$ such that $\Pi_{E} L v_{0}=e_{0}$. To see this, we consider a basis $\left\{e_{1}, \ldots, e_{m}\right\}$ of $E$. Then, $\Pi_{E} L v_{0}=e_{0}$ is equivalent to $\left(L v_{0}, e_{j}\right)=\left(v_{0}, L^{*} e_{j}\right)=\left(e_{j}, e_{0}\right)$ for $j=1, \ldots, m$.

This linear system of $m$ equations is solvable since $\left\{L^{*} e_{j}\right\}_{j=1, \ldots, m}$ are linearly independent.

Then, it is easy to observe that $v$ runs over the set where $\Pi_{E} L v=e_{0}$ if and only if $w=v-v_{0}$ runs over the set where $\Pi_{E} L w=0$ and that $L v$ is dense in $e_{0}+E^{\perp}$ if and only if $L w$ is dense in $E^{\perp}$.

To prove the lemma for $e_{0}=0$ we consider an element $f \in E^{\perp}$ such that

$(L v, f)=\left(v, L^{*} f\right)=0$ for all $v$ such that $\Pi_{E} L v=0$, i. e. $\left(v, L^{*} e_{j}\right)=$ 0 for $j=1, \ldots, m$ with the notations above. Then, there exist some 
coefficients $\alpha_{j}, j=1, \ldots, m$, such that $L^{*} f=\sum_{j=1}^{m} \alpha_{j} L^{*} e_{j}$, and since $L^{*}$ is injective $f=\sum_{j=1}^{m} \alpha_{j} e_{j}$. Therefore $f \in E$ and since $f \in E^{\perp}$ too, we deduce that $f=0$.

Collège de France

3 rue d'Ulm

75231 Paris Cedex 05. France

Departamento de Matemática Aplicada

Universidad Complutense

28040 Madrid. Spain

zuazuagsunma 4. mat . ucm.es

Recibido: 9 de Diciembre de 1996 